\title{
Onde termina o uso recreativo e inicia a dependência de jogos eletrônicos: uma revisão da literatura
}

\author{
Matheus Kereski Gonçalves \\ Luciana Schermann Azambuja
}

\begin{abstract}
Resumo: Os videogames ganharam destaque desde os anos 1970 e com a popularização da internet, originaram-se diversos relatos de que jovens vêm exagerando nos jogos fazendo com que em muitas vezes o uso deixe de ser recreativo. Esta pesquisa objetiva investigar, por meio da literatura, quando o uso de jogos eletrônicos é caracterizado como uma atividade recreativa e quando começa a ser considerado uma dependência. Foi realizada uma revisão na literatura na qual foram selecionados artigos científicos relacionados ao tema. Os resultados apontam que os jogos eletrônicos são a atividade de lazer mais praticada pelos adolescentes e por proporcionar um ambiente seguro e controlado permitem uma fuga da realidade para evitação dos problemas sociais. Concluindo, o uso abusivo dos jogos eletrônicos está associado a diversas comorbidades, interferindo de forma significativa na vida social, familiar, acadêmica e afetiva do sujeito, sendo que os adolescentes são os mais vulneráveis a desenvolver uma dependência.
\end{abstract}

Palavras Chave: Jogos Eletrônicos; Dependência; Adolescentes.

\section{Where recreational use ends and addiction to electronic games starts: a literature review}

\begin{abstract}
Video games have gained prominence since the 1970s and with the popularization of the internet, several reports have originated that young people have been exaggerating in games, often making their use stop being recreational. This research aims to investigate, through the literature, when the use of electronic games is characterized as a recreational activity and when it starts to be considered a dependency. A literature review was carried out in which scientific articles related to the topic were selected. The results show that electronic games are the leisure activity most practiced by teenagers and, by providing a safe and controlled environment, they allow an escape from reality to avoid social problems. In conclusion, the abusive use of electronic games is associated with several comorbidities, significantly interfering in the subject's social, family, academic and affective life, with adolescents being the most vulnerable to developing addiction.
\end{abstract}

Keywords: Electronic games; Dependency; Teens.

\section{Introdução}

Segundo Lima e Santos (2017), a evolução tecnológica permitiu uma mudança social, profissional e cultural na sociedade, de forma que o momento atual é marcado pelas relações virtuais. Os jogos eletrônicos cresceram juntamente com essa evolução e, são parte da cultura mundial (Gallo, 2007). Os videogames ganharam destaque nas décadas de 1970 e 1980 pela produção em grande escala e pela sua expansão, mas foi a partir da década de 1990 com os primeiros jogos on-line, que surgiu o interesse dos pesquisadores na identificação de um transtorno pelo uso abusivo ou dependência (Carvalho, Gonçalves, Brito \& Souza, 2018).

O termo "Jogo Eletrônico" segundo Pozzebon, Frigo e Oliveira (2014) remete a qualquer jogo que necessite da tecnologia de computadores, seja diretamente ou indiretamente, como, por exemplo, os videogames. Segundo Oliveira (2010), o jogo eletrônico pode ser definido como sendo uma atividade interativa que envolva regras e um contexto em que possa ser gerado um ambiente de interação social e que as ações dos jogadores possibilitem outras ações, até que um objetivo seja cumprido e, que a totalidade das ações supracitadas seja possível mediante um espaço, o qual é chamado virtual ou cibernético. Os jogos eletrônicos podem ser executados em computadores, videogames, celulares, algumas televisões entre outros dispositivos e têm como principal objetivo levar entretenimento aos seus usuários (Tavares, 2013).

De acordo com Ribeiro (2015), o primeiro jogo digital da qual se tem registro foi "Tennis for Two" de 1958, que para ser jogado precisava ser ligado a um osciloscópio ${ }^{1}$ conectado a um computador,

\footnotetext{
${ }^{1}$ Instrumento dentro de uma caixa usado na medida de sinais elétricos que apresenta gráficos em duas dimensões de um ou mais sinais elétricos, o eixo vertical (y) do monitor representa a intensidade da tensão e o eixo horizontal (x) representa o tempo, tornando o instrumento útil para mostrar sinais periódicos.
} 
sendo este muito diferente dos que existem nos dias de hoje. Lima e Santos (2017) afirmam que com o passar dos anos e com a evolução da tecnologia surgiram os primeiros vídeos games caseiros. Aparelhos eletrônicos com uma única e especifica função, a de rodar jogos, sendo que eram menores e mais baratos do que os computadores da época e compatíveis com qualquer aparelho de televisão. Console de jogos como o "Nintendo Entertainment System", e a indústria dos videojogos começaram a formar-se no final dos anos 1970. Nesse período, surgiram os primeiros dispositivos eletrônicos que ofereciam a experiência de jogo no conforto da casa, fazendo parte da vida e dos momentos de lazer das famílias (Freitas, 2015).

Com a chegada da internet e a facilidade para acessá-la, os jogos começaram a estar à disposição a qualquer hora, também por meio de aparelhos celulares, cada vez mais modernos, e em outras tecnologias eletrônicas (Lima \& Santos, 2017). Para Prioste (2013), embora as preferências por jogos virtuais apareçam mais comumente no início da adolescência, a formação do jogador inicia-se precocemente. $\mathrm{O}$ autor salienta que, os bebês já dispõem de brinquedos eletrônicos com telas que emitem sons quando são pressionadas. Com o avanço da tecnologia e com a propagação de aparelhos com jogos no mercado, desde videogames, tablets, videogames portáteis, notebooks, smartphones, vários aparelhos facilitam o acesso, tornando os jogos algo comum na infância e na adolescência (Lima \& Santos, 2017).

Abreu, Karam, Góes e Spritzer (2008) destacam que com a expansão do alcance da internet e com a popularização dos jogos eletrônicos, começaram a surgir relatos na imprensa e na literatura científica de sujeitos que estariam dependentes do mundo digital, da internet e dos jogos eletrônicos, e que muitas são as queixas de pais em consultórios psicológicos e psiquiátricos, preocupados com os filhos que estariam deixando de lado outras atividades da vida cotidiana, incluindo as tarefas escolares, o esporte e a convivência social. A dependência de jogos eletrônicos tem acometido crianças, adolescentes e jovens-adultos, ocasionando comorbidades que necessitam, cada vez mais, serem aprofundadas e tratadas por todas as áreas de Saúde Mental, o que inclui a Psicologia (Lemos \& Santana, 2012). Para Derevensky (2007), os adolescentes são os que mais apresentam problemas com a tecnologia e têm maior predisposição a riscos, maiores escores em medidas de impulsividade, de extroversão e de traços de ansiedade. Também apresentam maior nível de culpa, de ansiedade, uma menor estabilidade emocional, elevada baixa auto-estima, altas taxas de ideação suicida, de tentativas de suicídio e de depressão. Adolescentes do sexo masculino parecem ser mais vulneráveis a desenvolver hábitos problemáticos na prática de jogos eletrônicos (Lemos, Diniz, Peres \& Sougey, 2014).

Os jogos possibilitam aos usuários realizarem coisas que não podem ser feitas normalmente, em que o prazer de jogar se intensifica à medida que o jogador vivencia experiências anormais num ambiente seguro em que nada pode impedi-lo de alcançar o que deseja (Amaral, 2016). Os jogos eletrônicos passaram a fazer parte da vida cotidiana, sendo uma das principais atividades de lazer para crianças e adolescentes (Spritzer \& Picon, 2013). Para Amaral (2016), o jogo vem acompanhado de um sentimento de tensão e de alegria, juntamente com uma consciência de ser diferente da vida cotidiana. Os jogos são vistos como uma alternativa de socialização e colaboração para a maioria das pessoas (Suzuki, Matias, Silva \& Oliveira, 2009). Atualmente, as atividades recreativas tradicionais passaram a disputar espaço ou serem completamente substituídas pelo computador, televisão e os jogos eletrônicos. (Souza, Melo, Silva \& Silva, 2015).

Lemos e Santana (2012) identificaram a dependência de jogos eletrônicos como "possível transtorno psiquiátrico contemporâneo", embora reconhecessem que se tratava de um tipo de patologia, do qual o diagnóstico ainda estava em construção, havendo poucos estudos que o aprofundasse distintamente da dependência da internet e, em se tratando de estudos específicos acerca da dependência dos jogos eletrônicos e as formas de tratamento pela área da Psicologia, realizados no Brasil, são mais raros ainda. É de suma importância avanços nas explanações e estudos acerca do tema, a fim de evitar classificar os jogadores como dependentes. O DSM-5 (American Psychiatric Association [APA], 2014) menciona um novo transtorno, chamado transtorno do jogo pela internet, que se assemelha ao transtorno do jogo patológico (Souza, Boarolli, Jornada \& Gomes, 2016). Para que o indivíduo seja considerado dependente precisa ser incapaz de controlar a frequência e o tempo da atividade que antes era considerada inofensiva e acaba demonstrando excesso na prática (Retondar, Bonnet \& Harrys, 2016). Independentemente do diagnóstico, o sujeito que utiliza excessivamente o uso de tecnologias relata sofrimento e, por este motivo acabam solicitando ajuda orientação e tratamento (Fortim \& Araujo, 2013). Lima e Santos (2017) concluem dizendo que é preciso cuidado e que é necessário questionar se estamos frente a um verdadeiro "transtorno".

O presente trabalho tem como objetivo investigar por meio da literatura, quais os critérios necessários para que o jogo eletrônico seja considerado uma atividade recreativa e de lazer e quando começa a ser considerado uma dependência. Além disso, visa compreender os possíveis fatores associados ao uso excessivo dos jogos eletrônicos, bem como verificar as suas consequências. 


\section{Método}

Na elaboração desse trabalho foi realizado uma revisão de literatura sobre o tema proposto, visto que essa revisão tem como objetivo sumarizar as pesquisas já concluídas e obter conclusões a partir de um tema de interesse (Gonçalves, 2010). Após a escolha do tema, o pesquisador iniciou um amplo levantamento das fontes teóricas (artigos científicos), com o objetivo de elaborar a contextualização da pesquisa e seu embasamento teórico, o qual fez parte do referencial na forma de uma revisão bibliográfica (Prodanov \& Freitas, 2013). A revisão da literatura demonstra que o pesquisador está atualizado nas últimas discussões no campo de conhecimento em investigação.

A base de dados utilizada foi (Literatura Latino-Americana e do Caribe em Ciências da Saúde (LILACS), Scientific Electronic Library Online (ScIELO), PubMed, Periódicos Eletrônicos de Psicologia (PePSIC) e Google Acadêmico, serviram como instrumento de coleta de dados, a partir dos seguintes descritores: uso abusivo dos jogos eletrônicos, dependência de videogames, dependência de internet.

Quanto à amostra, os estudos foram selecionados a partir da variável de interesse, totalizando 38 publicações. A seleção foi realizada a partir de leitura criteriosa dos artigos, teses e dissertações encontradas nas bases de dados, sendo selecionada apenas a literatura que atendia aos critérios de inclusão definidos neste estudo. Foram incluídas apenas as publicações que responderam à questão do estudo, publicadas no período de 1998 a 2018, todos os tipos de delineamentos metodológicos foram aceitos. Posteriormente, foi realizada uma análise descritiva das mesmas, buscando estabelecer uma compreensão e ampliar o conhecimento sobre o tema pesquisado e elaborar o referencial teórico.

\section{Resultados e discussão}

Este estudo se propôs a investigar quais os critérios necessários para que o jogo eletrônico seja considerado uma atividade recreativa e de lazer e quando começa a ser considerado uma dependência. Diante disso, a literatura pesquisa apontou que os jogos eletrônicos são a maior atividade de lazer praticada por pessoas pelo mundo segundo Napoli e Gomes (2017). Com os avanços na área da tecnologia, para se ter acesso basta um smartphone, computador, tablet ou um console. No mercado de recreação com objetivo de divertimento o videogame foi o gerador de maior lucratividade no ano de 2014, com arrecadação de US\$60,4 bilhões anuais, valor maior que o adquirido com o cinema e a música (Meneses, 2015).

Segundo Suzuki et al. (2009), o uso de jogos eletrônicos é cada dia mais frequente como lazer, no desenvolvimento de habilidades e atividades que envolvam a aprendizagem $\mathrm{O}$ mercado de videogames continua a mostrar sua força e expansão por meio das novas tecnologias, de novos jogos, participando do mercado de smartphones, promovendo campeonatos e eventos para atrair antigos e novos consumidores (Ribeiro, 2015). A partir da criação dos primeiros videogames, pode se observar uma evolução nas práticas lúdicas dos jogos eletrônicos, desde a evolução dos recursos tecnológicos e a implementação da internet que proporcionou novas formas de interação social (Alves, 2005). Segundo Souza, Boarolli, Jornada e Gomes (2016), os videogames atualmente são os líderes no segmento da indústria digital e entretenimento para os adolescentes e jovens adultos. Os jogos eletrônicos são resultado das novas tecnologias e uma mídia contemporânea sendo que a sua dimensão atinge toda a sociedade de forma direta ou indireta, destacando-se neste meio as crianças, adolescentes e jovens adultos (Lemos \& Santana, 2012).

Não é possível falar dos jogos eletrônicos, sem antes sobre aqueles denominados jogadores, no Brasil a média de idade dos "gamers" é de 19 anos, diferentemente da média de idade dos jogadores americanos que é de 30 anos (Suzuki et al., 2009). Segundo Panayides e Walker (2012), a adolescência é um período crítico da vida, no qual ocorrem muitas mudanças nos fatores biológicos, sociais e psicológicos onde os adolescentes ficam mais suscetíveis a dependências. Para Ferreira e Sartes (2018), os jogadores adolescentes são os mais vulneráveis a desenvolver problemas com os jogos. Nesse sentido, Spritzer e Picon (2013), destacam que o uso excessivo dos jogos eletrônicos é um fenômeno global e atinge entre 5\% a $10 \%$ dos adolescentes, predominantemente do sexo masculino, os prejuízos mais observados nos dependentes de jogos eletrônicos são: baixo rendimento escolar, isolamento social e conflitos familiares. Para Fortim e Araújo (2013), os videogames podem ser usados para gerenciar um estado negativo do humor, e que isso pode ser um claro sinal de dependência. Meneses (2015) destaca que o Brasil possui a população que mais utiliza o seu tempo em ocupações online no mundo; em média o brasileiro passa 45 horas mensais (considerando o valor total de pessoas que possuem internet) passando ainda duas horas por dia jogando videogames. Segundo Suzuki et al. (2009), alguns jogadores chegam a jogar mais de 14 horas por dia, esquecendo a sua vida fora do jogo, e desistindo dos estudos e trabalho. Para Alves (2014), a dependência pode ser definida de forma geral como a tendência para fazer algumas 
atividades ou usar certas substâncias mesmo com as suas consequências que podem ser devastadoras sobre o bem estar social, físico, espiritual, psicológico e financeiro do sujeito. As dependências geralmente envolvem uma noção de mudança de hábito, de comportamento e repetição. Tanto as dependências comportamentais como as por abuso de substâncias geram uma dependência psicológica (Young, Yue \& Ying, 2011).

Spritzer e Picon (2013) destacam que os jogos são desenvolvidos para satisfazer as necessidades e desejos do ser humano e enfatizam quatro fatores dos jogos que causam a dependência: 1- Os jogos possuem uma narrativa envolvente, proporcionando ao jogador liberdade para criar o seu personagem, desta forma proporcionando uma grande imersão; 2- Manter um lugar seguro e que evite frustrar o jogador; 3- Valorizam as conquistas dos jogadores e baseiam o seu sistema em gratificações; 4- Interação com outros jogadores em um ambiente seguro proporcionados pela internet. Os jogos que promovem laços sociais e um ambiente confortável permitem uma fuga da realidade, que pode estar relacionada a problemas de ordem pessoal e emocional, o jogo acaba se tornando um mecanismo de fuga para escapar dos problemas sociais. Existem casos de pessoas que gastam muito dinheiro em jogos de smartphones e de videogames que vendem "facilidades" para finalizar missões dentro do jogo mais facilmente, desta forma "forçando" o jogador a fazer de tudo para completar determinado nível ou superar obstáculos e desafios, nem que para atingir esses objetivos ele precise gastar dinheiro real (Lima \& Santos, 2017).

Freitas (2015) afirma que têm sido comum os jogadores tornarem se dependentes dos jogos eletrônicos porque esses desejam: (i) desafios, pois eles querem ser desafiados a completar atividades, e sentir se mentalmente engajados na busca por soluções; (ii) socializar, porque eles desejam resolver os problemas dentro do jogo em grupo, com amigos ou familiares; (iii) experiências solitárias, nas quais eles possam ficar engajados dentro daquela experiência; (iv) gabar-se e ganhar o respeito dos outros jogadores, para isso enfatizam seus resultados e as suas conquistas; (v) recompensa emocional, experiências emocionais que envolvam tensão em conflitos, desespero em fracassos e euforia pelo sucesso; (vi) fantasiar, porque eles buscam o escapismo devido ao seu caráter imersivo e interativo e que permite a interpretação de outros personagens. Pozzebon et al. (2014) enfatizam que existem alguns registros de mortes causadas pela prática abusiva dos videogames, por exemplo, um jogador que ficou 23 horas seguidas participando de partidas de League of Legends, faleceu devido a um ataque cardíaco. No ano de 2008, o número de jogadores na China foi de 59 milhões e o número de dependentes em jogos eletrônicos na Coréia do Sul foi estimado entre 300.000 a 500.000 (Meneses, 2015).

Além do objetivo geral, o estudo visou compreender os possíveis fatores associados ao uso excessivo dos jogos eletrônicos, bem como verificar as suas consequências. Nesse sentido Prioste (2013), destaca que as demandas de tratamento psicológico na adolescência estão ligadas à compulsão por jogos online, deixam de lado outras atividades da vida cotidiana, incluindo as tarefas escolares, o esporte e a convivência social. $\mathrm{O}$ avanço da tecnologia proporcionou que os jogos eletrônicos invadissem o cotidiano das pessoas, estando presente em TVs, smartphones, videogames, computadores e demais aparelhos, que apresentam facilidade e conforto para as pessoas (Cavalli, Trevisol \& Vendrame, 2013). De acordo com Meneses (2015), o smartphone por ser um aparelho móvel com acesso à internet muda a visão da população de que um viciado em jogos deveria estar em frente a um personal computer (PC), tendo em vista que a internet pode estar na "palma da mão". A evolução tecnológica proporciona cada vez mais uma imersão maior nos jogos, num universo em que estes apresentam mais qualidade técnica como: realismo gráfico, sons de alta qualidade, interatividade e velocidade dos jogos (Spritzer \& Picon, 2013).

Abreu et al. (2008) afirmam que a dependência em jogos eletrônicos pode ser considerada um comportamento desadaptativo, em que o jogador passa a não mais controlar a frequência de tempo jogado, passando de um comportamento que anteriormente era inofensivo para um comportamento desadaptativo e que entre os dependentes há uma vulnerabilidade pessoal prévia, baixa tolerância à frustração, alta esquiva, ansiedade e baixa auto-estima. Segundo Spritzer e Picon (2013), a maioria dos dependentes de jogos eletrônicos apresenta alguma comorbidade psiquiátrica, como: problemas sociais, depressão, ansiedade social e déficit de atenção e hiperatividade (TDAH). O uso abusivo dos jogos acaba levando o indivíduo a uma serie de prejuízos como: fracasso escolar, dificuldade de se relacionar, aumento de peso e isolamento social. De acordo com Souza et al. (2016), a dependência em jogos pode ocasionar transtornos comórbidos, como a ansiedade, a depressão, entre outros. Na pesquisa realizada por Suzuki et al. (2009) foram identificados jogadores com ansiedade, depressão, fobias e TDA. Quanto às comorbidades, Abreu et al. (2008) destacam a depressão, o transtorno de humor bipolar, os transtornos de ansiedade e o TDAH. Diversas comorbidades estão associadas ao uso abusivo dos jogos eletrônicos, dentre elas a obesidade, baixo rendimento escolar e a violência (Ferreira \& Sartes, 2018).

Embora se tenham dados registrados acerca do uso abusivo dos jogos eletrônicos e do reconhecimento do comportamento de dependência, bem como de comorbidades que essa disfunção ou 
"transtorno" tem ocasionado nas pessoas, Abreu et al. (2008), chamam a atenção para a dificuldade que ainda existe em ser estabelecido um diagnóstico e mesmo assim, também tem provindo de outros tipos de dependências, como a de substância e da internet, que se tem notícias dos esforços em contextualizar a dependência em jogos eletrônicos. O maior problema não é o uso dos jogos, mas a perda de controle desse mecanismo, que passa a tornar esse comportamento padrão, sendo uma estratégia de escape e evitação (Fortim \& Araujo, 2013).

Conti et al. (2012) referem que houve uma primeira tentativa, em 1995, pelo psiquiatra americano Ivan Gildberg em definir o uso abusivo da internet, mas foi em 1996 que Kimberly S. Young se utilizou dos critérios indicados pelo DSM-IV para Dependência de Substâncias para apurar um conceito de dependência da internet (e, nela, também nos jogos eletrônicos). Os critérios diagnósticos propostos por Young (1998) para a Dependência da Internet foram os seguintes: 1. Preocupação excessiva com a internet; 2 . Necessidade de aumentar o tempo conectado (on-line) para ter a mesma satisfação; 3. Exibir esforços repetidos para diminuir o tempo de uso da internet; 4. Presença de irritabilidade e/ou depressão; 5. Quando o uso da internet é restringido, apresenta labilidade emocional (internet como forma de regulação emocional); 6. Permanecer mais conectado (on-line) do que o programado; 7. Trabalho e relações sociais em risco pelo uso excessivo; 8. Mentir aos outros a respeito da quantidade de horas on-line (Abreu et al., 2008). Para ser estabelecido um diagnóstico de dependência da internet, reconhecida pela expressão "Internet Addiction" era preciso reconhecer cinco ou mais dos critérios (Young, 1998).

Griffiths (2005) definiu as seguintes características centrais que deveriam ser identificadas nos usuários da internet que para serem considerados dependentes dos jogos eletrônicos: Saliência: quando o jogo se torna a atividade mais importante da vida do indivíduo, dominando seus pensamentos e comportamentos; modificação de humor/euforia: experiência subjetiva de prazer, euforia ou mesmo alívio da ansiedade relatada pelo jogador; Tolerância: necessidade de jogar por períodos cada vez maiores para atingir a mesma modificação de humor; Abstinência: estados emocionais e físicos desconfortáveis quando ocorre descontinuação ou redução súbita do jogo (intencional ou forçada); Conflito: pode ser entre o jogador e as pessoas próximas (conflito interpessoal), conflito com outras atividades (trabalho, escola, vida social, prática de esportes, etc.) ou mesmo do indivíduo com ele mesmo relacionado ao fato de estar jogando excessivamente; Recaída/estabelecimento: tendência de retornar rapidamente ao padrão anterior de jogo excessivo após períodos de abstinência ou controle.

Esses critérios com o passar do tempo, foram vistos como "periféricos", "genéricos", e se por um tempo serviu à Psicologia e/ou a outras áreas terapêuticas para identificar os dependentes de jogos eletrônicos, com o tempo sucessivas pesquisas foram sendo empreendidas visando explicar o fenômeno da impulsividade, compulsões pelos jogos eletrônicos (Lemos \& Santana, 2012). Segundo Riego (2018) o termo "distúrbio de games", ou compulsão por games que foi incluído na CID 11 estuda a relação do videogame com as possíveis incapacidades de interação do indivíduo na sociedade tendo como base outros distúrbios já incluídos no CID 11; todos com conexão aos videogames como transtorno do jogo pela internet ou da dependência de internet. De acordo com a Organização Pan-Americana de Saúde e Organização Mundial de Saúde (OPAS/OMS, 2018):

A décima primeira versão da Classificação Internacional de Doenças (CID-11) entrará em vigor em $1^{\circ}$ de janeiro de 2022. Essa versão é uma pré-visualização e permitirá aos países planejar seu uso, preparar traduções e treinar profissionais de saúde. Descrito como Gaming Disorder (Distúrbio por jogos), a condição foi inclusa no rascunho do CID-11 em 2018. Os critérios de diagnóstico adotados pela CID-11 são: Controle prejudicado sobre jogos (início, frequência, intensidade, duração, término, contexto); Maior prioridade dada aos jogos, ao ponto que o jogo teria uma procedência sobre outros aspectos da vida e atividades diárias; Atividade contínua nos jogos apesar da ocorrência de consequências negativas (OPAS/OMS, 2018). Conforme a OPAS/OMS (2018), para um possível diagnóstico, o indivíduo deve ser observado por um período de 12 meses (Riego, 2018). Segundo Souza et al. (2016), o Manual Diagnóstico e Estatístico de Transtornos Mentais (DSM-5) menciona um novo transtorno, chamado Transtorno do Jogo pela Internet, que se assemelha ao transtorno do jogo patológico, no qual a área da Psicologia tem se pautado para analisar os sintomas da dependência por jogos eletrônicos e decidir pelo tratamento. De acordo com Lemos, Conti e Sougey (2015), o DSM-5 propõe os seguintes critérios diagnósticos para o Transtorno do Jogo pela Internet: 1. Preocupação com jogos eletrônicos, tornando-se a atividade dominante na vida diária; 2. Sintomas de abstinência quando os jogos são retirados (irritabilidade, ansiedade ou tristeza, mas sem sinais físicos de abstinência farmacológica); 3. Necessidade de despender cada vez mais tempo jogando; 4. Tentativas frustradas de controlar a participação nos jogos; 5 . Perda de interesse em antigos passatempos e entretenimentos; 6 . Uso excessivo continuado de jogos pela internet, apesar do conhecimento dos problemas psicossociais; 7. Enganar a 
família, terapeutas ou outros quanto ao tempo despendido com os jogos; 8. Uso dos jogos para evitar ou aliviar o humor negativo; 9. Colocar em risco ou perder relacionamentos, emprego ou oportunidade educacional ou de carreira devido à participação em jogos pela internet (APA, 2014).

Cabe registrar-se que Lima e Santos (2017) concluíram o seu estudo dizendo que "[...] é preciso cuidado e questionar se estamos frente a um verdadeiro 'transtorno'. É preciso cuidado e de mais estudos acerca do tema a fim de evitar classificar os jogadores como dependentes”.

\section{Considerações finais}

As novas tecnologias vêm causando um impacto significativo no mundo contemporâneo e os jogos eletrônicos são hoje a atividade de lazer mais praticada entre os adolescentes. Sendo assim esta pesquisa teve como objetivo investigar por meio da literatura quando o uso de jogos eletrônicos é caracterizado como uma atividade recreativa e de lazer e quando começa a ser considerado uma dependência. Visando compreender as possíveis causas do uso excessivo dos jogos eletrônicos, bem como verificar as suas consequências.

Em relação à dependência em jogos eletrônicos o uso recreativo passa a ser considerado uma dependência quando percebem-se prejuízos e o seu uso deixa de ser um comportamento controlado, passando a ser uma preocupação na vida do sujeito, onde rotinas, responsabilidades e relacionamentos passam a ser corrompidos ou ignorados pela necessidade de se manter jogando.

A partir do presente estudo constata-se que a maioria dos usuários dependentes apresenta alguma vulnerabilidade pessoal como, por exemplo, baixa tolerância a frustrações, ansiedade, depressão, transtorno de déficit de atenção, dentre outras. E que ouso abusivo pode acarretar prejuízos na vida do sujeito, como: baixo rendimento escolar, isolamento social e problemas familiares. A adolescência potencializa aos jovens serem os alvos mais propensos a dependência, por ser um período da vida marcado pela formação de identidade, e pela busca constante de sua auto-afirmação. E os jogos eletrônicos por proporcionar uma imersão em mundos de fantasias, da qual permite que os jogadores exerçam o controle das situações apresentadas e acabe gerando um mundo sem frustrações e rejeições, desta forma os usuários vão se afastando do mundo real e substituindo pelo mundo virtual, como um mecanismo de fuga dos problemas sociais.

Sendo assim, este estudo visa contribuir e estimular mais pesquisas com essa temática, para que seja um assunto mais debatido e pesquisado na sociedade. Indica-se que novos e atualizados estudos sejam realizados de forma interdisciplinar, do qual a Psicologia poderá contribuir, eficientemente para uma melhor forma de tratamento da dependência de jogos eletrônicos.

\section{Referências}

Abreu, C. N., Karam, R. G., Góes, D. S., \& Spritzer, D. T. (2008). Dependência de Internet e de jogos eletrônicos: uma revisão. Revista Brasileira de Psiquiatria 30(2), 156-67.

Alves, L. (2005). Game over: jogos eletrônicos e violência. São Paulo: Futura.

Alves, G. M. (2008). A Construção da Identidade do Adolescente e a Influência dos Rótulos na mesma. Dissertação Curso de Psicologia, Universidade do Extremo Sul Catarinense, SC, Brasil.

Alves, P. A. B. A. (2014). Dependência da Internet Efeitos na Saúde. Dissertação de mestrado, Instituto Superior de Engenharia de Coimbra, Coimbra, Portugal.

Amaral, A. C. S. (2016). Consumo infantil: análise sobre a influência dos jogos eletrônicos na vida das crianças. Trabalho de conclusão de curso, Universidade Federal do Rio Grande do Sul, Porto Alegre, RS, Brasil.

American Psychiatric Association. (2014). DSM-5: Manual diagnóstico e estatístico de transtornos mentais. Porto Alegre: Artmed.

Carvalho, H. S., Gonçalves, F. S., Brito, V. S., \& Souza, E. (2018). Validação da escala pvp para avaliar o uso problemático de videojogos em adolescentes. Rev. Elet Estácio Saúde, 7(1), 20-25.

Cavalli, F.S., Trevisol, M. T., \& Vendrame, T. (2013). Influência dos jogos eletrônicos e virtuais no comportamento social dos adolescentes. Psicol. Argum., 31(72), 155-163.

Conti, M. A., Jardim, A. J., Hearst, N., Cordás, T. A., Tavares, H., \& Abreu, C. N. (2012). Avaliação da equivalência semântica e consistência interna de uma versão em português do Internet Addiction Test (IAT). Rev. Psiq. Clín., 39(3), 106-10.

Derevensky, J. L. (2007). Impulsividade: Jogo entre jovens e jogo problemático. In Gigliotti, A., Guimarães (Ed.), A Dependência, compulsão e impulsividade (Cap. 11, pp. 81 - 90). Rio de Janeiro: Editora Rubio.

Ferreira, M. B. O., \& Sartes, L. M. A. (2018). Uma Abordagem Cognitivo-Comportamental do Uso Prejudicial de Jogos Eletrônicos. Gerais: Revista Interinstitucional de Psicologia, 11(2), 306-326. 
Fortim, I., \& Araújo, C. A. (2013). Aspectos psicológicos do uso patológico de internet. Tese de Doutorado em Psicologia Clínica, Pontifícia Universidade Católica de São Paulo. SP, Brasil.

Freitas, C. C. (2015). Jogo pervasivo para crianças: a convergência dos meios físico e digital. Trabalho de conclusão de Curso, Universidade Federal do Rio Grande do Sul. Porto Alegre, RS, Brasil.

Gallo, S. N. (2007). Jogo como elemento da cultura: aspectos contemporâneos e as modificações na experiência de jogar. Tese de doutorado. Pontifícia Universidade Católica de São Paulo, SP, Brasil.

Gonçalves, L.S.V. (2010). A família e o portador de transtorno mental: Estabelecendo um vínculo para a reinserção à sociedade. Trabalho de Conclusão de Curso. Universidade Federal de Minas Gerais, Manhuaçu, MG, Brasil.

Griffiths, M. D. (2005). A "components" model of addiction within a biopsychosocial framework. Journal of Substance Use, 10, 191-197.

Lemos, I. L., \& Santana, S. M. (2012). Dependência de jogos eletrônicos: a possibilidade de um novo diagnóstico psiquiátrico. Rev. psiquiatr. clín., 39(1), 28-33.

Lemos, I. L., Diniz, P. R. B., Peres, J. F. P., \& Sougey, E. B. (2014). Neuroimagem na dependência de jogos eletrônicos: uma revisão sistemática. Jornal Brasileiro de Psiquiatria, 63(1), 57-71.

Lemos, I. L., Conti, M. A., \& Sougey, E. B. (2015). Avaliação da equivalência semântica e consistência interna da Game AddictionScale (GAS): versão em português. J. bras. psiquiatr, 64(1), 8-16.

Lima, A. L., \& Santos, A. A. (2017). Videogames e a sua influência no comportamento das pessoas. ELocução Revista Iniciação Científica da FAEX, 1(12), 40-45.

Meneses, G, P. (2015). Videogame é droga? Controvérsias em torno da dependência de jogos eletrônicos. Dissertação de Mestrado, Universidade de São Paulo, SP, Brasil.

Napoli, F. D., \& Gomes, F. G. C. (2017). Jogos virtuais violentos e influências no psiquismo humano. Revista Uningá Review, 29(1), 196-203.

Oliveira, Y. G. (2010). Jogos eletrônicos como ferramenta de ensino. Trabalho de Conclusão de Curso. Universidade Federal do Rio de janeiro, RJ, Brasil.

Organização Pan-Americana de Saúde / Organização Mundial de Saúde. Brasil (2018). OMS divulga nova Classificação Internacional de Doenças (CID 11). OPAS/OMS. Recuperado em 20 de março de 2019, de <https://www.paho.org/bra/index.php?option=com_content\&view=article\&id=5702:oms-divulganova-classificacao-internacional-de-doencas-cid-11\&Itemid=875> .

Panayides, P., \& Walker, M. J. (2012). Evaluation of the Psychometric Properties of the Internet Addiction Test (IAT) in a Sample of Cypriot High School Students: The Rasch Measurement Perspective. Europe's Journal of Psychology, 8(3), 327-351.

Pozzebon, E., Frigo, L., \& Oliveira, L. (2014). Perfil dos jogadores brasileiros de MMO -Massively Multiplayer Online Game. Trabalho de Conclusão de Curso. Universidade Federal de Santa Catarina, SC, Brasil.

Prioste, C. D. (2013). O adolescente e a internet: laços e embaraços no mundo virtual. Tese de Doutorado, Universidade de São Paulo, SP, Brasil.

Prodanov, C. C., \& Freitas, E. C. (2013). Metodologia do trabalho científico: métodos e técnicas da pesquisa e do trabalho acadêmico ( $2^{\mathrm{a}}$. Ed). Novo Hamburgo: Feevale.

Retondar, J. J. M., Bonnet, J. C., \& Harris, E. R. A. (2016). Jogos eletrônicos: corporeidade, violência e compulsividade. Revista Brasileira de Ciências do Esporte 38(1), 3-10.

Ribeiro, A. C. M. (2015). A indústria de vídeo games e seu impacto econômico. Trabalho de Conclusão de Curso. Universidade Federal do Mato Grosso, Mato Grosso, Brasil.

Riego, B. L. P. (2018). Aficionado por vídeo games ou jogadores compulsivos? Trabalho de Conclusão de Curso. Centro Universitário UNIFAAT, São Paulo, SP, Brasil.

Souza, C. A. M., Melo, J. M., Silva, W. M., \& Silva, C. L. (2015). Os Significados Dos Jogos Eletrônicos Para Jovens De Uma Escola Técnica De Campinas-Sp. Revista Brasileira de Estudos do Lazer, 2(1), 44-63.

Souza, B.D., Boarolli, M., Jornada, L. K., \& Gomes, K. M. (2016). Vício em jogos eletrônicos (play addiction) em adolescentes. Revista Iniciação Científica, 14(1), 19-28.

Spritzer, D. T., \& Picon, F. (2013). Dependência de jogos eletrônicos. In: Abreu,C. N., Eisenstein, E., Estefenon, S. G. Vivendo esse mundo digital:impactos na saúde, na educação $e$ nos comportamentos sociais. Porto Alegre: Artmed.

Suzuki, F. T. I., Matias, M. V., Silva, M. T. A., \& Oliveira, M. P. M. T. (2009). O uso de videogames, jogos de computador e internet por uma amostra de universitários da Universidade de São Paulo. $J$ Bras Psiquiatr., 58(3), 162-168. 
Tavares, G. C. (2013). O Uso Abusivo de Jogos Eletrônicos: Definição e Tratamento. Trabalho de Conclusão de Curso. Universidade Luterana do Brasil, Santa Maria, RS, Brasil.

Young, K. S. (1998). Internet addiction: the emergence of a new clinical disorder. Cyberpsychol Behav., 1(3), 237-44.

Young, K. S., Yue, X. D., \& Ying, L. (2011). Dependência de Internet: Manual e Guia de Avaliação e Tratamento. Porto Alegre: Artmed.

Matheus Kereski Gonçalves: Acadêmico do curso de psicologia pela Universidade Luterana do Brasil (ULBRA) - Campus São Jerônimo - Antônio de Carvalho, s/nº Esquina com RS 401 - RS - CEP 96700000. Telefone: (51) 999380550.

Luciana Schermann Azambuja: Professora Orientadora. Doutora em Medicina e Ciências da Saúde pela Pontifica Universidade Católica do Rio Grande do Sul. Neuropsicologa do Centro Clínico da PUCRS. Av Ipiranga, 6690/314. POA/RS. Telefone: (51) 33205067.]

E-mail para contato: matheus17.gon@gmail.com 